\title{
Avaliação da adição da epinefrina à bupivacaína intra-articular no controle da dor aguda em pacientes submetidos a reconstrução do ligamento cruzado anterior*
}

\section{Assessment of the Addition of Epinephrine to Intra-articular Bupivacaine for the Control of Acute Pain in Patients underwent to Anterior Cruciate Ligament Reconstruction}

\author{
Marcos George de Souza Leão ${ }^{10} \quad$ Juscimar Carneiro Nunes ${ }^{20}$ Ivan Tramujas da Costa e Silva ${ }^{2}$ \\ Alan Braga Perfeito ${ }^{10}$ Wagner de Paula Rogério ${ }^{3 \text { () }}$ Rafaela Brasil e Silva Nunes ${ }^{3(0)}$ \\ ${ }^{1}$ Departamento de Ortopedia e Traumatologia, Fundação Hospital \\ Adriano Jorge, Manaus, AM, Brasil \\ 2 Programa de Pós-Graduação em Cirurgia, Universidade Federal do \\ Amazonas, Manaus, AM, Brasil \\ Endereço para correspondência Marcos George de Souza Leão, MD, \\ MSc, Departamento de Ortopedia e Traumatologia, Fundação \\ Hospital Adriano Jorge, Avenida Carvalho Leal, 1.778, Cachoeirinha, \\ Manaus, AM, 69065-001, Brasil (e-mail: mgsleao@uol.com.br).
}

${ }^{3}$ Serviço de Anestesiologia, Fundação Hospital Adriano Jorge, Manaus, AM, Brasil

Rev Bras Ortop 2022;57(2):257-266

\begin{abstract}
Resumo

Resumo

Palavras-chave
- ligamento cruzado
anterior
- medição da dor
- analgesia
- tratamento
farmacológico
- artroscopia

Objetivo Avaliar primariamente a dor pós-operatória, por meio da escala visual analógica (EVA), nos pacientes submetidos a reconstrução do ligamento cruzado anterior (RLCA) que receberam soluções anestésicas intra-articulares (IAs).

Métodos Ensaio clínico randomizado com uma amostra de 48 pacientes, divididos em 4 grupos: Grupo I $(\mathrm{n}=12)$ - $20 \mathrm{ml}$ de solução fisiológica (controle); Grupo II $(\mathrm{n}=12)-20 \mathrm{ml}$ de bupivacaína a $0,5 \%$; Grupo III $(\mathrm{n}=12)-20 \mathrm{ml}$ de bupivacaína a $0,5 \%+0,1 \mathrm{mg}$ de epinefrina; e Grupo IV $(\mathrm{n}=12)-20 \mathrm{ml}$ de solução fisiológica $+0,1 \mathrm{mg}$ de epinefrina, injetados no joelho ao término da cirurgia. A dor foi avaliada pela EVA imediatamente e 6, 12, 24 e 48 horas após o procedimento.

Resultados Observou-se grande variabilidade nos resultados da EVA entre os pacientes avaliados em cada grupo. Verificou-se, pela análise de variância (analysis of variance, ANOVA) de Kruskal-Wallis, considerando um nível de 5\% de significância, que as soluções anestésicas IAs de cada grupo influenciaram na avaliação da dor desses pacientes $(p=0,003)$, sendo os do Grupo III os que apresentaram menor dor pósoperatória. Não se evidenciou um maior ou menor consumo de drogas analgésicas suplementares, ou efeitos adversos das decorrentes das soluções empregadas.
\end{abstract}

Trabalho desenvolvido no Departamento de Ortopedia e Traumatologia, Fundação Hospital Adriano Jorge, Cachoeirinha, Manaus, AM, Brasil.

recebido

29 de Agosto de 2019

aceito

06 de Julho de 2020

published online

Abril 19, 2021
DOI https://doi.org/ 10.1055/s-0040-1718514. ISSN 0102-3616. (c) 2021. Sociedade Brasileira de Ortopedia e Traumatologia. All rights reserved.

This is an open access article published by Thieme under the terms of the Creative Commons Attribution-NonDerivative-NonCommercial-License, permitting copying and reproduction so long as the original work is given appropriate credit. Contents may not be used for commercial purposes, or adapted, remixed, transformed or built upon. (https://creativecommons.org/ licenses/by-nc-nd/4.0/)

Thieme Revinter Publicações Ltda., Rua do Matoso 170, Rio de Janeiro, RJ, CEP 20270-135, Brazil 


\section{Abstract}

\section{Keywords}

- anterior cruciate ligament

- pain assessment

- analgesia

- drug therapy

- arthroscopy
Conclusão A solução combinada de bupivacaína e epinefrina foi a mais eficaz no controle da dor nos pacientes submetidos a RLCA, mas sem diferenças estatisticamente significativas com relação ao grupo II $(p=0,547)$. Não se observou diminuição ou aumento no consumo de analgésicos suplementares, ou o aparecimento de efeitos sistêmicos adversos $(p>0,05)$.

Objective To evaluate postoperative pain, using the visual analog scale (VAS), in patients undergoing anterior cruciate ligament reconstruction (ACLR) and receiving intra-articular anesthetic solutions.

Methods The present is a randomized clinical trial with a sample of 48 patients divided into 4 groups: Group I $(n=12)-20 \mathrm{~mL}$ of saline solution (control); Group II $(\mathrm{n}=12)-20 \mathrm{~mL}$ of $0.5 \%$ bupivacaine; Group III $(\mathrm{n}=12)-20 \mathrm{~mL}$ of $0.5 \%$ bupivacaine $+0.1 \mathrm{mg}$ of epinephrine; and Group IV $(n=12)-20 \mathrm{~mL}$ of saline solution $+0.1 \mathrm{mg}$ of epinephrine. These solutions were injected into the knee at the end of the surgery. Pain was assessed using the VAS immediately and 6, 12, 24 and 48 hours after the procedure.

Results The VAS scores were highly variable among the groups. A Kruskal-Wallis analysis of variance (ANOVA), considering a level of significance of $5 \%$, revealed that all intra-articular anesthetic solutions influenced the assessment of pain $(p=0.003)$, and that Group-III subjects presented less postoperative pain. There was no evidence of a higher or lower use of supplemental analgesic agents, or of adverse effects resulting from these anesthetic solutions.

Conclusion Bupivacaine combined with epinephrine was the most effective solution for pain control in patients undergoing ACLR, but with no statistically significant differences when compared to Group II $(p=0.547)$. There was no decrease or increase in the use of supplemental analgesics or in the occurrence of adverse systemic effects $(p>0.05)$.

\section{Introdução}

O alívio da dor pós-operatória continua a ser um dos principais desafios médicos. Apesar dos grandes avanços na compreensão da fisiopatologia da dor aguda e do desenvolvimento de novos medicamentos analgésicos e de técnicas de administração de fármacos, um número significativo de pacientes continua a ter dor pós-operatória de difícil controle. ${ }^{1}$

Os anestésicos locais intra-articulares (IAs) são muitas vezes utilizados para a prevenção da dor aguda após a cirurgia artroscópica do joelho; no entanto, a magnitude desta dor varia de paciente para paciente. Em um esforço para encontrar o método ideal de analgesia pós-operatória eficaz e de longa duração, muitos fármacos diferentes, incluindo opioides, antiinflamatórios não esteroides (AINEs), cetamina, clonidina e neostigmina, foram adicionados às soluções anestésicas IAs, ${ }^{2,3}$ não existindo consenso e padronização acerca das doses e de quais fármacos devam ser utilizados.

A bupivacaína é frequentemente utilizada para analgesia IA por causa do seu período ativo e eficácia. A sua eficácia, especialmente em administração única, foi estudada porque seu efeito sobre a dor pós-operatória é conceitualmente simples. ${ }^{4}$

A maneira mais frequente de se avaliar a dor é por meio da Escala Visual Analógica (EVA), um instrumento que tenta aferir uma característica ou atitude que se acredita que varia ao longo de uma série contínua de valores, e que não pode ser facilmente medida diretamente. Também é útil para analisar se o tratamento está sendo eficaz, quais procedimentos têm surtido melhores resultados, assim como se há alguma deficiência no tratamento de acordo com o grau de melhora ou piora da dor. A quantidade de dor sentida pelo paciente varia ao longo de uma faixa que vai de nenhuma dor (zero) à dor extrema (dez), conforme descrito por Wewers e Lowe. ${ }^{5}$

O objetivo primário deste trabalho foi avaliar, por meio da EVA, a dor pós-operatória aguda nos pacientes submetidos a reconstrução do ligamento cruzado anterior (RLCA) que receberam soluções anestésicas IAs e, secundariamente, descobrir qual solução anestésica é mais eficaz no controle da dor, e observar em quais períodos a dor foi mais bem controlada, bem como observar os efeitos adversos e a necessidade de analgesia suplementar.

\section{Métodos}

Foi feito um ensaio clínico randomizado, triplo cego, em quatro grupos de pacientes com diagnóstico de instabilidade crônica do joelho com indicação de tratamento cirúrgico, cujo tamanho foi estimado em relação ao total de pacientes atendidos na instituição, sendo de 3,33\% ao mês. Foi 
estabelecido um nível de $95 \%$ de confiança, e uma precisão (margem de erro) de 5\%, no que resultou em uma amostra de 48 pacientes.

Na amostra, foram incluídos pacientes: com diagnóstico de instabilidade crônica do joelho; maiores de 18 anos; com classificação do estado físico de acordo com a escala da American Society of Anesthesiologists (ASA) I e II; sem doenças inflamatórias locais e sistêmicas; com lesões isoladas do LCA (sem outros ligamentos), sem histórico de fraturas ou cirurgias prévias na região do joelho; em cuja cirurgia foram utilizados dos tendões flexores mediais como enxerto; e operados pelo pesquisador principal e sua equipe. Como critérios de exclusão, consideraram-se: pacientes esqueleticamente imaturos; lesões condrais maiores do que $2 \mathrm{~cm}^{2}$; procedimentos cirúrgicos associados, tais como osteotomias e outros ligamentos; pacientes sem condições clínicas, hipertensos, com coagulopatias, grávidas; uso crônico de anticoagulantes; uso de analgésicos até $24 \mathrm{~h}$ antes do procedimento cirúrgico; pacientes que se recusaram a assinar o termo de consentimento livre e esclarecido (TCLE); pacientes não operados na instituição; a não aceitação do anestesiologista em seguir o protocolo do projeto; e aqueles pacientes que se autodeclararam indígenas.

Foi confeccionado um instrumento de coleta de dados próprio para esta pesquisa, e se anotaram os dados pertinentes aos pacientes e à cirurgia, tais como: nome, peso, idade e registro, data, o lado operado, o grupo ao qual o paciente pertencia (I, II, III ou IV), o tempo de isquemia durante a cirurgia, e a frequência cardíaca $(F C)$ e a pressão arterial (PA) imediatamente ao término da cirurgia (T0), 6 horas após a cirurgia (T1), 12 horas após a cirurgia (T2), 24 horas após a cirurgia (T3), e 48 horas após a cirurgia (T4).
Foram descritos os possíveis efeitos adversos, bem como a necessidade de analgesia suplementar. A EVA colorida com os respectivos horários da coleta dos dados foi adicionada. Lesões associadas (meniscais e condrais) foram anotadas, da mesma forma que o diâmetro dos túneis ósseos no fêmur e na tíbia para a reconstrução ligamentar.

As diretrizes dos Padrões Consolidados para o Relato de Ensaios Clínicos (Consolidated Standards of Reporting Trials, CONSORT) foram seguidas para a realização do presente estudo. ${ }^{6}$

Os pacientes foram alocados seguindo o resultado da randomização; foi solicitada a autorização de internação hospitalar para que os pacientes fossem encaminhados à cirurgia conforme a vaga e a fila de espera dos pacientes.

Utilizando-se o software livre disponível no website www.randomization.com, foram distribuídos aleatoriamente 48 pacientes em 12 blocos de 4 grupos iguais, em que cada grupo recebeu uma solução IA no joelho conforme a alocação de acordo com o que se segue (-Figura 1):

Grupo I $(\mathrm{n}=12): 20 \mathrm{ml}$ de solução fisiológica (SF) a 0,9\% (controle);

Grupo II $(\mathrm{n}=12): 20 \mathrm{ml}$ de bupivacaína a 0,5\%;

Grupo III $(\mathrm{n}=12): 20 \mathrm{ml}$ de bupivacaína a 0,5\% + 0,1 mg de epinefrina; e

Grupo IV $(n=12): 20 \mathrm{ml}$ de SF a $0,9 \%+0,1 \mathrm{mg}$ de epinefrina.

Um dos fatores que provocam a dor no pós-operatório imediato é a distensão capsular pelo sangramento; então, teorizou-se que, diminuindo o sangramento, poderia haver diminuição da dor, e, por esse motivo, os pacientes dos grupos III e IV receberam a epinefrina associada ou isolada.

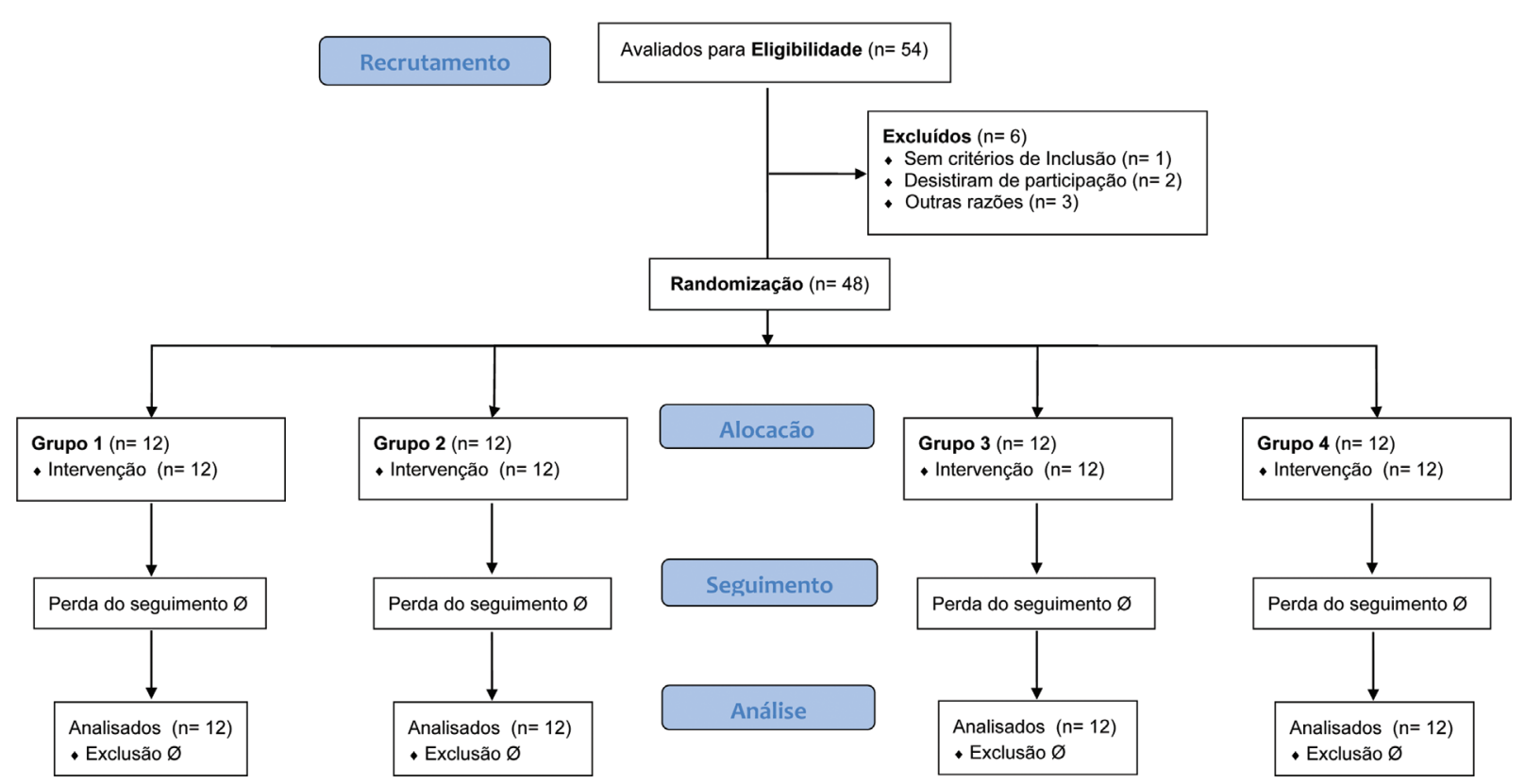

Fig. 1 Fluxograma CONSORT. 
O preparo da solução sempre foi realizado por um membro auxiliar da pesquisa (médico residente de anestesiologia, que não tinha contato com o paciente antes e após cirurgia) cerca de 20 minutos antes do término da cirurgia, por comunicação direta do autor pesquisador. $\mathrm{O}$ cirurgião não tinha conhecimento do conteúdo das soluções a serem injetadas.

Os pacientes foram anestesiados por meio de bloqueio subaracnoide com bupivacaína hiperbárica a $0,5 \%$ (dose de $15 \mathrm{a} 20 \mathrm{mg}$ ) associada a $20 \mathrm{mcg}$ de fentanil, e não foram administradas outras modalidades de analgesia multimodal, a fim de controlar o máximo possível as variáveis relativas à dor e à analgesia no período pós-operatório.

Todas as cirurgias foram realizadas pela via artroscópica, com acesso articular por meio dos portais anterolateral (AL) alto e anteromedial (AM), sendo os túneis femoral e tibial perfurados na posição anatômica, no centro da cicatriz do LCA prévio, via transportal AM para passagem do enxerto e sua posterior fixação. Em todos os pacientes, a reconatrução do LCA foi feita com o uso dos tendões dos músculos flexores mediais do joelho - semitendíneo (ST) e grácil (G) ipsilaterais -, coletados mediante incisão sobre a pata de ganso no terço proximal da perna, com aproximadamente $3 \mathrm{~cm}$ de comprimento, antes da artroscopia propriamente dita; as lesões meniscais eram tratadas por meniscectomia parcial ou subtotal.

Após a confecção das suturas da pele, as soluções foram injetadas no joelho pelo portal AL, de acordo com a alocação dos grupos. Terminadas as suturas e o com curativo compressivo confeccionado, o torniquete pneumático era desinsuflado.

A intensidade da dor foi avaliada pela EVA (— Figura 2), sendo o estímulo doloroso causado pela flexão do joelho a $45^{\circ}$ repetido por três vezes, e medido com o auxílio do goniômetro em T0, T1, T2, T3 e T4, por um médico que não tinha conhecimento dos grupos estudados. A necessidade de complementação analgésica foi anotada no instrumento de coleta de dados: com AINEs por via intravenosa (IV; $40 \mathrm{mg}$ de tenoxicam 1 vez ao dia), com opioides ( $50 \mathrm{mg}$ de tramadol de 6 em 6 horas, e $10 \mathrm{mg}$ de morfina em solução IV lenta até de 4 em 4 horas).

Durante a internação, os pacientes receberam analgesia com dipirona na dose de $1 \mathrm{~g} \mathrm{IV}$, a intervalos de 4 horas de maneira sistemática, realizaram tromboprofilaxia com $40 \mathrm{mg}$ de enoxaparina sódica 1 vez ao dia, pela via subcutânea por 10 dias, e crioterapia foi aplicada na região anterior do joelho por 20 minutos de 4 em 4 horas. A deambulação precoce e a movimentação foram estimuladas e realizadas conforme a tolerância individual, com auxílio de fisioterapeuta. Os pacientes, caso as condições clínicas permitissem, recebiam alta hospitalar, o que ocorria geral- mente após $48 \mathrm{~h}$ do procedimento cirúrgico, sendo neste período coletados os últimos dados da pesquisa, pois esta é a rotina do serviço.

Após o estímulo doloroso, foram anotadas a FC e a PA. Também foram anotados os efeitos adversos sistêmicos das soluções e medicamentos, tais como sudorese, tremores, náuseas e vômitos, hipotensão, prurido, retenção urinária com necessidade de sondagem de alívio, taquicardia ou bradicardia, erupção cutânea e cefaleia, se porventura ocorressem.

Após o estímulo doloroso, foram anotadas a FC e a PA. Também foram anotados os efeitos adversos sistêmicos das soluções e medicamentos, tais como sudorese, tremores, náuseas e vômitos, hipotensão, prurido, retenção urinária com necessidade de sondagem de alívio, taquicardia ou bradicardia, erupção cutânea e cefaleia, se porventura ocorressem.

Foi realizada a distribuição de frequência absoluta (n) e relativa (\%) dos dados enumerados (atributos ou dados nominais) e a estatística descritiva dos dados quantitativos (grandezas específicas ou variáveis). Para as comparações entre as amostras de parâmetros nominais, utilizou-se o teste do Qui-quadrado, ou, na impossibilidade de seu uso, o teste exato de Fisher. Realizou-se uma análise descritiva dos dados, e os resultados foram apresentados em tabelas de contingência (atributos) ou de estatística (grandezas), com distribuição de frequência, gráficos e medidas descritivas. Para avaliar a influência da analgesia IA entre os grupos, realizou-se a análise de variância (analysis of variance, ANOVA). Para a tomada de decisão em todas as relações entre as variáveis e os testes de hipóteses, adotou-se o nível de significância de $5 \%(p=0,05)$.

Neste estudo, foi utilizada a técnica ANOVA. Para a comparação entre as médias, utilizou-se o teste de Tukey. Foi incluído o teste de Kruskal-Wallis, um método não paramétrico usado para testar se um conjunto de amostras provém da mesma distribuição, sendo uma extensão do Teste de Mann-Whitney para mais de duas amostras.

Os dados foram tabulados em planilhas do Programa Microsoft Excel 2016 (Microsoft Corp., Redmond, WA, EUA), e analisados pelo programa estatístico Minitab (Minitab, LLC, State College, PA, EUA), versão 14.1.

O projeto foi submetido ao Comitê de Ética em Pesquisa (CEP) da instituição, com o número de Certificado de Apresentação para Apreciação Ética - CAAE: 50651315.8.0000.0007, e recebeu o parecer consubstanciado com o número 1.774 .789 em 13 de outubro de 2016, e foi também aprovado no Clinical Trials, com o registro RBR - 7PCKQT. Todos os pacientes que porventura concordassem participar da pesquisa assinavam o TCLE.

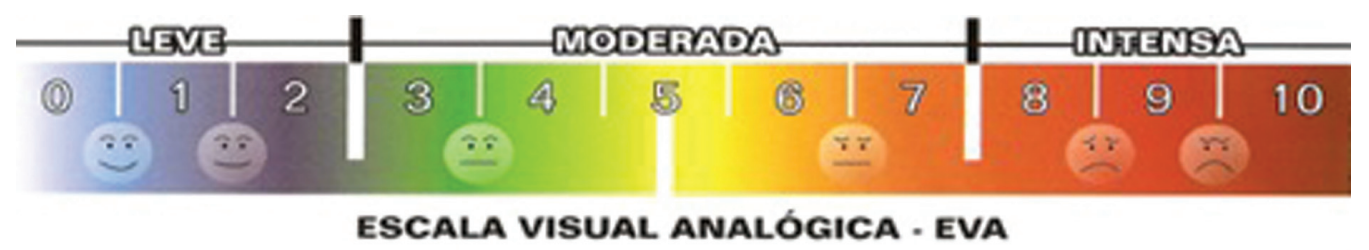

Fig. 2 Escala Visual Analógica da dor. 
Tabela 1 Características clínicas dos pacientes avaliados

\begin{tabular}{|l|l|l|}
\hline CARACTERÍsTICAS & $\begin{array}{l}\text { FREQUÊNCIA } \\
(\mathbf{n}=\mathbf{4 8})\end{array}$ & $\%$ \\
\hline Lado & & \\
\hline Esquerdo & 18 & 37,5 \\
\hline Direito & 30 & 62,5 \\
\hline Tempo de Isquemia (min) & & \\
\hline 75 a 85 & 9 & 18,8 \\
\hline 86 a 96 & 6 & 12,5 \\
\hline 97 a 107 & 16 & 33,3 \\
\hline 108 a 118 & 11 & 22,9 \\
\hline$>118$ & 6 & 12,5 \\
\hline Túnel & & \\
\hline Sete & 6 & 12,5 \\
\hline Oito & 34 & 70,8 \\
\hline Nove & 8 & 16,7 \\
\hline Lesões & & \\
\hline Presente & 31 & 64,6 \\
\hline Ausente & 17 & 35,4 \\
\hline Tipo de Lesão & $(\mathrm{n}=38)$ & \\
\hline Lesão associada & 1 & 2,6 \\
\hline Lesão condral troclear & 1 & 2,6 \\
\hline $\begin{array}{l}\text { Sequela de fratura } \\
\text { na espinha tibial }\end{array}$ & 1 & 2,6 \\
\hline Menisco lateral & 14 & 36,8 \\
\hline Menisco lateral & 21 & 55,3 \\
\hline
\end{tabular}

\section{Resultados}

Dos pacientes analisados, $36(75,0 \%)$ eram do gênero masculino, e 12 (25,0\%), do gênero feminino. A idade variou entre 18 e 54 anos, com média de $31 \pm 10$ anos (desvio padrão, DP). Os pacientes da amostra selecionada tinham idade mediana de 33 anos. Em relação à lateralidade dos joelhos, 18 pacientes (37,5\%) foram submetidos a cirurgia do joelho esquerdo, e 30 (62,5\%), foram submetidos a cirurgia do joelho direito. 0 tempo de isquemia variou entre 75 e 120 minutos, com tempo médio de 102,02 $\pm 15,02$ minutos (-Tabela $\mathbf{1}$ ).

Havia presença de lesões associadas em 31 (64,6\%) pacientes, e ausência dessas lesões em 17 (35,4\%) pacientes. Nesses 31 pacientes, foram encontradas 38 lesões, incluindo uma lesão condral troclear e uma lesão condral (lesão associada) no côndilo femoral medial, sendo a lesão do menisco medial a mais frequente entre as observadas ( - Tabela $\mathbf{1}$ ).

A dor pós-operatória foi avaliada pela EVA, e está descrita na -Tabela 2.

Observando apenas os períodos de tempo na classificação da EVA dos pacientes avaliados, tem-se que, no pós-operatório imediato (T0), 47 (97,9\%) pacientes estavam com dor leve, e 01 (2,1\%), com dor moderada. No momento T1, 18 (37,5\%) pacientes estavam com dor leve, 27 (56,3\%), com dor moderada, e 03 (6,3\%), com dor intensa. No momento T2, 15 $(31,3 \%)$ pacientes estavam com dor leve, 31 (64,6\%), com dor moderada, e $2(4,2 \%)$, com dor intensa. No momento T3, 16 $(33,3 \%)$ pacientes estavam com dor leve, 30 (62,5\%), com dor moderada, e 02 (4,2\%), com dor intensa. E, no momento T4, 20 $(41,7 \%)$ pacientes estavam com dor leve, $27(56,3 \%)$, com dor moderada, e 01, (2,1\%), com dor intensa.

Considerando um nível de $5 \%$ de significância, verificouse, pela ANOVA de Kruskal-Wallis, que as soluções anestésicas IAs de cada grupo influenciaram na avaliação da dor desses pacientes $(p=0,003)$ ( - Tabela 3 ).

Observou-se que a ANOVA da dor dos pacientes sofreu forte influência tanto da analgesia IA estabelecida em cada grupo $(p<0,0001)$ quanto do intervalo de tempo fixado na análise experimental $(p<0,0001)$. Na interação do tempo com a analgesia IA estabelecida nos grupos, não houve diferenças significativas $(p<0,286)$ ( - Tabela 4).

Para encontrar o momento que mais causou a variabilidade da dor dos pacientes, foi realizada a análise das comparações múltiplas pelo teste de Tukey. Desse modo, observou-se que a variabilidade maior ocorreu entre o T0 e todos os outros momentos do experimento $(p<0,0001)$.

Ao realizar as comparações múltiplas das médias de dor entre os grupos, observou-se que houve diferenças significativas entre as médias de dor do Grupo I em comparação com o Grupo III ( $p=0,014)$, e entre as médias do Grupo II com o Grupo IV $(p=0,042)$. Ocorreu ainda diferença significativa entre as médias do Grupo III com o Grupo IV $(p=0,001)$ (-Tabela 5).

Quanto aos efeitos adversos, encontrou-se a sua presença em $2(4,2 \%)$ pacientes, e nos $46(95,8 \%)$ restantes não houve ocorrência de efeitos. Dos 2 pacientes que tiveram efeitos adversos, um era do Grupo I e teve náuseas, e o outro era do Grupo 3 e teve cefaleia.

Quanto à utilização da analgesia coadjuvante, 7 pacientes $(14,6 \%)$ fizeram uso desse tipo de analgesia complementar, sendo que 6 fizeram uso de opioides, e 1 fez uso de opioides e anti-inflamatório. Os outros 41 pacientes (85,4\%) não necessitaram de analgesia adicional.

Para comprovar que a analgesia coadjuvante não havia se tornado um fator de confundimento experimental, verificouse se havia relação entre o uso das soluções IAs e o uso de analgesia coadjuvante. Sendo assim, concluiu-se que, pelo teste do Qui-Quadrado de Pearson, no nível de 5\% de significância, não houve relação significativa entre as soluções e o uso da analgesia complementar $(p=0,606)$ ( - Tabela 6 ).

\section{Discussão}

O sucesso de qualquer intervenção médica deve ser julgado, inclusive de acordo com as percepções dos pacientes, em relação aos benefícios obtidos com o tratamento, seja ele conservador ou cirúrgico. A busca de uma analgesia ideal para uso após artroscopia nas RLCAs tornou-se cada vez mais importante com um maior número de cirurgias sendo realizadas.

A RLCA é associada com dor pós-operatória significativa, conforme demonstrado neste trabalho, o que pode limitar a 
Tabela 2 Análise descritiva da dor pós-operatória dos pacientes avaliados

\begin{tabular}{|c|c|c|c|c|c|c|c|}
\hline TEMPO & GRUPO & $\mathbf{n}$ & MÉDIA & Desvio padrão & MÍNIMO & MEDIANA & MÁXIMO \\
\hline \multirow[t]{4}{*}{ Pós-operatório imediato (T0) } & 1 & 12 & 0,0 & 0,0 & 0,0 & 0,0 & 0,0 \\
\hline & II & 12 & 0,3 & 0,9 & 0,0 & 0,0 & 3,0 \\
\hline & III & 12 & 0,0 & 0,0 & 0,0 & 0,0 & 0,0 \\
\hline & IV & 12 & 0,1 & 0,3 & 0,0 & 0,0 & 1,0 \\
\hline \multirow[t]{4}{*}{06 horas de pós-operatório (T1) } & I & 12 & 5,1 & 1,2 & 3,0 & 5,5 & 6,0 \\
\hline & II & 12 & 3,1 & 2,1 & 1,0 & 2,0 & 8,0 \\
\hline & III & 12 & 2,4 & 2,2 & 0,0 & 1,0 & 6,0 \\
\hline & IV & 12 & 4,8 & 2,4 & 2,0 & 4,5 & 9,0 \\
\hline \multirow[t]{4}{*}{12 horas de pós-operatório (T2) } & I & 12 & 4,4 & 2,5 & 0,0 & 6,0 & 7,0 \\
\hline & II & 12 & 3,6 & 2,5 & 0,0 & 3,0 & 7,0 \\
\hline & III & 12 & 2,8 & 1,7 & 0,0 & 2,5 & 5,0 \\
\hline & IV & 12 & 5,3 & 2,3 & 1,0 & 5,5 & 9,0 \\
\hline \multirow[t]{4}{*}{24 horas de pós-operatório (T3) } & 1 & 12 & 4,3 & 2,6 & 0,0 & 5,0 & 7,0 \\
\hline & II & 12 & 3,3 & 2,1 & 0,0 & 3,0 & 7,0 \\
\hline & III & 12 & 2,6 & 2,3 & 0,0 & 2,0 & 7,0 \\
\hline & IV & 12 & 4,8 & 2,5 & 1,0 & 4,5 & 9,0 \\
\hline \multirow[t]{4}{*}{48 horas de pós-operatório (T4) } & I & 12 & 3,6 & 2,4 & 0,0 & 4,5 & 7,0 \\
\hline & II & 12 & 2,8 & 1,9 & 0,0 & 2,5 & 6,0 \\
\hline & III & 12 & 2,7 & 2,8 & 0,0 & 2,5 & 8,0 \\
\hline & IV & 12 & 3,8 & 2,5 & 0,0 & 3,5 & 7,0 \\
\hline
\end{tabular}

Tabela 3 Análise da variância da dor dos pacientes operados

\begin{tabular}{|l|l|l|l|l|}
\hline GRUPO & $\mathbf{n}$ & MEDIANA & RANK & Valor de $\boldsymbol{p}$ \\
\hline I & 12 & 4,50 & 133,6 & \multirow{2}{*}{0,003} \\
\cline { 1 - 3 } II & 12 & 2,00 & 112,5 & \\
\cline { 1 - 3 } III & 12 & 1,50 & 97,1 & \\
\cline { 1 - 3 } IV & 12 & 4,00 & 138,8 & \\
\cline { 1 - 3 } & &
\end{tabular}

Nota: Análise de variância de Kruskal Wallis.

possibilidade de realizar este procedimento em pacientes ambulatoriais (alta hospitalar no mesmo dia - day hospital). Em nosso serviço, a cirurgia ainda é realizada com internação tradicional, e a alta hospitalar é dada com 24 a 48 horas, devido ao inadequado controle da dor e até por motivos culturais. Muitos serviços, inclusive no Brasil, realizam este procedimento ambulatorialmente, e, nos Estados Unidos, cirurgias mais complexas, como próteses totais de joelho e quadril, têm sido realizadas em regime ambulatorial num grupo específico de pacientes, o que demonstra o sucesso no controle da dor no pós-operatório. Alguns dos nossos pacientes, inclusive, apresentam dor pós-operatória de intensidade 9, medida pela EVA, e todos receberam alta em 48 horas após a cirurgia. Dificuldades na adaptação dos métodos comuns de tratamento da dor pós-operatória em pacientes hospitalizados para pacientes ambulatoriais resultaram em um manejo inadequado da dor após a cirurgia, e, neste estudo, mesmo nos pacientes nos quais foi realizada a infiltração IA de bupivacaína, houve casos em que se atingiu o nível de dor 8, pela EVA, após 48 horas do procedimento, o que revela a necessidade de um controle álgico satisfatório.

Tabela 4 Análise da variância da dor pela Escala Visual Analógica dos pacientes internados

\begin{tabular}{|l|l|l|l|l|}
\hline CAUSA DA VARIAÇÃO & GRAUS DE LIBERDADE & SOMA DOS QUADRADOS & QUADRADO MÉDIO & Valor de $\boldsymbol{p}$ \\
\hline Tempo $^{1}$ & 4 & 17,23 & 17,2333 & 0,000 \\
\hline Grupo $^{2}$ & 3 & 4,37 & 4,3667 & 0,000 \\
\hline Tempo versus grupo & 12 & 3,30 & 3,3 & 0,286 \\
\hline Resíduo & 220 & 50,50 & 50,5 & \\
\hline TOTAL & 239 & 75,40 & & \\
\hline
\end{tabular}

Notas: ${ }^{1} 6,12,24$ e 48 horas após a cirurgia. ${ }^{2}$ I, II, III e IV. 
Tabela 5 Comparações múltiplas das médias de dor entre os grupos

\begin{tabular}{|l|l|l|l|l|l|}
\hline Tukey & & & & & \\
\hline \multirow{2}{*}{ GRUPO } & \multirow{2}{*}{ MODELO PADRÃO } & \multirow{2}{*}{ Valor de $\boldsymbol{p}$} & \multicolumn{2}{|l|}{ INTERVALO DE CONFIANÇA DE 95\% } \\
\cline { 4 - 6 } & & & Limite inferior & Limite superior \\
\hline \multirow{4}{*}{ Grupo I } & Grupo II & 0,088 & 0,323 & $-0,08$ & 0,38 \\
\cline { 2 - 6 } & Grupo III & 0,088 & 0,014 & 0,04 & 0,49 \\
\cline { 2 - 6 } & Grupo IV & 0,088 & 0,779 & $-0,31$ & 0,14 \\
\hline \multirow{4}{*}{ Grupo II } & Grupo I & 0,088 & 0,323 & $-0,38$ & 0,08 \\
\cline { 2 - 6 } & Grupo III & 0,088 & 0,547 & $-0,11$ & 0,34 \\
\cline { 2 - 6 } & Grupo IV & 0,088 & 0,042 & $-0,46$ & $-0,01$ \\
\hline \multirow{4}{*}{ Grupo III } & Grupo I & 0,088 & 0,014 & $-0,49$ & $-0,04$ \\
\cline { 2 - 6 } & Grupo II & 0,088 & 0,547 & $-0,34$ & 0,11 \\
\cline { 2 - 6 } & Grupo IV & 0,088 & 0,001 & $-0,58$ & $-0,12$ \\
\hline \multirow{3}{*}{ Grupo IV } & Grupo I & 0,088 & 0,779 & $-0,14$ & 0,31 \\
\cline { 2 - 6 } & Grupo II & 0,088 & 0,042 & 0,01 & 0,46 \\
\cline { 2 - 6 } & Grupo III & 0,088 & 0,001 & 0,12 & 0,58 \\
\hline
\end{tabular}

Tabela 6 Relação entre as soluções intra-articulares e o uso da analgesia suplementar

\begin{tabular}{|l|l|l|l|l|l|}
\hline \multirow{2}{*}{ GRUPO } & \multicolumn{4}{l|}{$\begin{array}{l}\text { USO DE ANALGESIA COADJU- } \\
\text { VANTE }\end{array}$} & \multirow{2}{*}{ Total } \\
\cline { 2 - 5 } & Sim & $\%$ & Não & $\%$ & \\
\hline I & 3 & 25,0 & 9 & 75,0 & 12 \\
\hline II & 1 & 8,3 & 11 & 91,7 & 12 \\
\hline III & 1 & 8,3 & 11 & 91,7 & 12 \\
\hline IV & 2 & 16,7 & 10 & 83,3 & 12 \\
\hline TOTAL & 7 & $\mathbf{1 4 , 6}$ & $\mathbf{4 1}$ & $\mathbf{8 5 , 4}$ & $\mathbf{4 8}$ \\
\hline
\end{tabular}

Nota: Teste do Qui-quadrado: $p=0,606$.

A RLCA é considerada um procedimento altamente bemsucedido no campo da medicina desportiva; no entanto, o manejo da dor pós-operatória nessas cirurgias ainda não foi bem alcançado de forma adequada. Apesar de o nível da dor em geral que ocorre após a RLCA não ter sido associado com dor intolerável, a cirurgia pode levar a considerável desconforto durante o período pós-operatório imediato. Além disso, relatou-se que uma dor significativa após o procedimento cirúrgico teve um efeito negativo sobre a evolução e causou a insatisfação do paciente, ${ }^{7}$ sendo estes dados corroborados no nosso trabalho, no qual a necessidade de analgesia suplementar foi mínima, mas, no Grupo II (bupivacaína a 0,5\% isolada), houve pacientes com nível de dor até 8 medido pela EVA.

Osborne e Keene ${ }^{8}$ não conseguiram provar a eficácia do uso de bupivacaína a 0,5\% associada ou não com 0,2 mg de epinefrina, quando comparado ao uso de placebo. É provável que os fatores pré-operatórios, tais como o desconforto, a tolerância à dor aguda ou crônica, e patologias de quaisquer natureza sejam os determinantes mais importantes na dor pós-operatória, ou seja, o status pré-operatório do joelho é o melhor preditor da evolução pós-operatória. No entanto, essas variáveis não foram analisadas no presente estudo, apesar de ele ter desenho e resultados semelhantes ao do estudo citado.

Existem relatos ${ }^{9}$ que evidenciam que a adição de morfina e cetorolaco à ropivacaína IA aumenta a eficácia analgésica do anestésico local, reduz o consumo de analgésicos após a alta hospitalar, e melhora alguns aspectos das atividades da vida diária (incapacidade de dormir por causa da dor, apetite, concentração, necessidade de assistência, capacidade de caminhar em terreno plano sem dor, capacidade de ir para o trabalho) sem aumentar os efeitos colaterais após a cirurgia. Como não havia disponibilidade de cetorolaco e ropivacaína na instituição onde o presente trabalho foi realizado, eles não foram utilizados, e a morfina IV era administrada em caso de dor intensa e insuportável, ou com EVA $>6$. Não foi objeto da nossa pesquisa verificar a quantidade de analgésicos consumidos após a alta hospitalar; os desfechos foram verificados até 48 horas após a cirurgia.

Uma variável impactante nas injeções IAs é o uso de epinefrina. ${ }^{10}$ Seu uso também tem sido recomendado para prevenir a toxicidade do anestésico local. A bupivacaína IA é injetada ao final do procedimento, e se habilita como um bom analgésico; além disso, a sua ação sistêmica carece de maiores efeitos adversos. Isso foi bem demonstrado neste estudo, no qual a analgesia nas primeiras 12 horas foi superior no grupo III (bupivacaína e epinefrina), que tinha níveis de EVA inferiores em relação aos outros grupos.

Neste estudo, o tramadol foi utilizado por via IV apenas em caso de dor moderada (EVA $>5$ ), diferentemente do que foi relatado no estudo de Zeidan et al., ${ }^{11}$ no qual a mistura IA de $100 \mathrm{mg}$ de tramadol com bupivacaína a 0,25\% diminuiu a pontuação na EVA, com melhor analgesia pós-operatória do que a produzida por injeção IA de um ou outro medicamentos isolados. Esta solução também esteve associada com a recuperação mais rápida da deambulação sem apoio e com a alta hospitalar mais precoce. 0 tramadol foi prescrito para 
cinco pacientes, mas não o associamos à uma diminuição da percepção da EVA.

Kristensen et al. ${ }^{12}$ mostram que a analgesia com infiltração local com ropivacaína e epinefrina e o bloqueio do nervo femoral (BNF) são semelhantes na gestão da dor pós-operatória após a RLCA com enxerto dos tendões flexores mediais do joelho. Até estudos randomizados investigarem o BNF combinado com a infiltração no sítio doador, os autores recomendam a analgesia com infiltração local na RLCA com enxerto dos isquiotibiais mediais do joelho.

No presente trabalho, houve necessidade de analgesia suplementar em apenas 7 pacientes (14,1\%), o que demonstra que a analgesia IA é útil na redução da incapacidade dos pacientes no pós-operatório. Ela previne o aparecimento da dor, e ajuda a evitar a necessidade de drogas adicionais. Normalmente, é possível atingir uma boa analgesia no pósoperatório imediato com a administração (IV, oral) de drogas analgésicas. No entanto, essas substâncias nem sempre atingem o objetivo por serem inespecíficas, e podem trazer consigo muitos efeitos colaterais, tais como lesões gástricas agudas. A combinação de $150 \mathrm{mg}$ de ropivacaína com $30 \mathrm{mg}$ de cetorolaco injetados IA ao final da cirurgia artroscópica do joelho aumenta a eficácia analgésica dos anestésicos locais sem aumentar os efeitos colaterais; além disso, houve mais efeito sedativo neste grupo de 7 pacientes. ${ }^{13} \mathrm{Na}$ presente investigação, a presença de efeitos adversos foi verificada em apenas dois pacientes e esses efeitos foram considerados leves (cefaleia e náusea), não sendo verificada nenhuma correlação com os grupos ou medicamentos estudados.

No estudo de Jazayeri et al., ${ }^{14}$ o efeito analgésico pósoperatório da injeção IA de morfina e tramadol após pequenas cirurgias artroscópicas no joelho foi máximo 6 horas após a injeção. Os efeitos analgésicos observados na avaliação da presente pesquisa foram mais bem observados nas primeiras 6 horas, sem o inconveniente dos efeitos adversos dos opioides; portanto, o controle da dor foi mais eficiente nas primeiras 12 horas, principalmente nas primeiras 6 horas.

Os anestésicos locais que contêm epinefrina podem causar condrotoxicidade significativa em condrócitos humanos em modelos in vitro. A epinefrina isolada, a uma concentração de 1:100.000 e 1:200.000, não teve nenhum efeito sobre a viabilidade dos condrócitos, bem como o conservante utilizado: metilparabeno. ${ }^{15}$ Por isso, optamos por utilizar a epinefrina em concentração de 1:200.000.

A administração IA de bupivacaína e/ou morfina não teve efeito analgésico forte o suficiente para explicar seu uso frequente em pacientes submetidos a RLCA, com enxerto de tendões flexores, sob anestesia raquidiana, ${ }^{16}$ apesar da tendência a menos dor em todos os momentos do grupo III (bupivacaína e morfina), o índice de dor pela EVA foi baixo em todos os grupos e momentos; a dor foi controlada com medicações mais simples e de baixo custo. Os nossos resultados mostraram maior eficácia analgésica com a solução de bupivacaína isolada e a combinada de bupivacaína e epinefrina, quando comparadas com placebo (SF).

Observamos menores valores da EVA em todos os momentos estudados do Grupo III, principalmente nas primeiras 24 horas após a cirurgia, diferente do relatado (em outra referência nacional) por Souza et al., ${ }^{17}$ que não encontraram diferenças na analgesia pós-operatória quando utilizados a morfina, a bupivacaína, o fentanil e o SF IA para cirurgia artroscópica do joelho sob anestesia subaracnóidea, na maioria dos períodos investigados (imediatamente após a cirurgia e de 6 em 6 horas até as 24 horas). Na sexta hora após a cirurgia, o grupo do fentanil apresentou intensidade da dor significativamente menor, e o grupo da morfina necessitou de mais complementação analgésica.

Na literatura, a utilização de anestesia subaracnóide para RLCA foi associada a náuseas e vômitos pós-operatórios, e a profilaxia combinada com dexametasona e perfenazina foi associada com menos efeitos adversos em pacientes que foram anestesiados desta maneira. ${ }^{18}$ No presente estudo, as náuseas (01 paciente) não foram achado frequente, e não se observaram casos de vômitos. De acordo com a técnica anestésica, todos recebem dexametasona na indução, com o intuito de evitar tais complicações.

No presente estudo, não houve correlação entre o aumento do consumo de opiodes e os efeitos colaterais das drogas utilizadas e a natureza do grupo estudado. Dal et al. ${ }^{19}$ observaram redução da dor pós-operatória e consumo adequado de drogas analgésicas com o emprego de cetamina IA, da bupivacaína, ou a utilização de neostigmina. Esses autores não observaram quaisquer efeitos secundários psicomiméticos, particularmente aqueles observados com doses mais elevadas ou o uso sistêmico, e a administração IA de cetamina promoveu analgesia duradoura e eficaz, semelhante à neostigmina, mas menos eficaz do que a bupivacaína após a artroscopia do joelho, sem quaisquer efeitos adversos.

Todos os grupos que receberam as soluções, fossem elas combinadas ou não, mostraram diminuição nas pontuações de dor aferidas pela EVA, sendo os resultados semelhantes (apesar do desenho e do uso de drogas diferentes) aos de um estudo clínico prospectivo, randomizado e duplo-cego; 20 isso sugere que, na artroscopia do joelho, as injeções analgésicas combinadas consistindo de morfina, bupivacaína, epinefrina e bupivacaína associada à epinefrina resultaram em menores níveis de dor e diminuição do consumo de narcóticos na sala de recuperação anestésica, com diferenças estatisticamente significativas quando comparadas com epinefrina sozinha. Estes resultados foram independentes do momento da injeção, fosse ela pré- ou pós-operatória.

O uso das soluções no Grupo III permitiu um controle analgésico satisfatório em pacientes submetidos a RLCA com anestesia raquidiana. No Brasil, o bloqueio espinhal é de prática corriqueira, e a alta hospitalar é dada habitualmente no dia seguinte ou com 48 horas, como no presente trabalho. A literatura internacional é abundante em descrições de analgesia em pacientes submetidos a RLCA com anestesia geral e BNF com alta hospitalar frequentemente no mesmo dia. Em pacientes submetidos a artroscopia do joelho e não a RLCA, em que o estímulo doloroso é maior, e sob anestesia espinhal, Eroglu et al. ${ }^{21}$ verificaram níveis de dor menores com a associação de $5 \mathrm{mg}$ de morfina e $20 \mathrm{ml}$ de bupivacaína IA a 0,25\%, quando comparados à administração placebo (SF).

Mesmo quando comparamos no presente estudo a bupivacaína isolada (Grupo II) com placebo e até a epinefrina 
isolada (Grupo IV), verificamos uma analgesia superior, com achados semelhantes aos de Wei et al.,22 o que demonstra que uma dose única IA de bupivacaína evidenciou ser significativamente melhor do que o placebo no alívio da dor após a cirurgia artroscópica do joelho. Mais ensaios clínicos randomizados e controlados, de alta qualidade e com longo tempo de seguimento, são altamente necessários para determinar a segurança da dose única de bupivacaína. Mesmo assim, a utilização rotineira da dose única IA de bupivacaína é uma forma eficaz de controlar a dor após a cirurgia artroscópica do joelho. Em suma, não se pode chegar à conclusão de que uma dose única IA de bupivacaína é tóxica ou segura clinicamente.

Recentemente, Zhou et al., ${ }^{23}$ em uma metanálise, concluíram que uma dose única de ropivacaína IA administrada ao final da cirurgia artroscópica do joelho proporciona alívio eficaz da dor nos períodos pós-operatório imediato e precoce, sem aumentar os efeitos colaterais de curto prazo. Como não dispúnhamos da ropivacaína, lançamos mão da bupivacaína, que tem mecanismo de ação semelhante, e chegamos a resultados de analgesia similares.

Iwasaki et al. ${ }^{24}$ demonstraram não haver fortes evidências de que a injeção IA de bupivacaína induza alterações degenerativas na cartilagem articular, sem diferenças na viabilidade celular, densidade celular ou pontuações de avaliação da cartilagem; portanto, os resultados podem se aplicar às articulações normais e osteoartríticas.

Esta pesquisa apresenta algumas limitações. O tempo decorrido entre a lesão e a cirurgia não foi levado em consideração, pois o status pré-operatório do joelho pode modificar a percepção da dor pelos pacientes, e, quanto maior o tempo decorrido da lesão, pior o joelho estará. Não se determinou se os pacientes continuaram com o mesmo nível de atividade. A coleta de dados, com o objetivo de captar com mais sensibilidade o padrão de dor, poderia ter sido feita com intervalos mais curtos. A dor poderia ter sido estratificada por gênero e idade, a fim de se coletarem informações mais precisas sobre esse assunto, mais isso fugiria dos objetivos assinalados. Também existem outras escalas de aferição da dor, mas, pela facilidade de adoção e compreensão por parte dos pacientes, foi adotada a EVA. $O$ status mental dos pacientes também pode modificar a percepção dolorosa, mas não foi objetivo deste estudo. Com toda a polêmica em torno do uso de anestésicos locais IA, no tocante à condrotoxidade, esta só poderia ser verificada com um seguimento longo e mediante estudo histológico nos pacientes, que teriam de ser submetidos a novo procedimento cirúrgico. $O$ fato de coexistirem outras lesões IA (meniscal e condral, que envolvem a tróclea e o côndilo femoral medial) pode proporcionar um fator a mais na gênese da dor, além da RLCA per se, mas também não foi observada uma relação entre os grupos estudados e lesões associadas. O sítio doador do enxerto é potencial fonte de dor, sendo sugestão para trabalhos posteriores. Os efeitos sistêmicos adversos dos medicamentos foram anotados pelo corpo de enfermagem, e podem ter sido subnotificados. $\mathrm{O}$ uso da analgesia coadjuvante pode causar confundimento, pois a dor pode melhorar tanto pela solução quanto pela analgesia, ocasionando um viés de confusão. Existem outras potenciais maneiras de controle da dor, tais como BNF e bloqueio do canal do adutor, mas esses procedimentos não são realizados rotineiramente no nosso serviço.

\section{Conclusão}

De acordo com os achados do presente estudo, concluiu-se que a epinefrina não acrescenta benefício na redução do processo de dor pós-operatória; independente da presença do anestésico IA, o controle da dor é melhor em períodos mais curtos do pós-operatório (até 12 horas); a presença ou não de epinefrina e/ou bupivacaína no espaço IA não induz a presença de efeitos adversos sistêmicos; não houve diferença quantitativa na utilização adjuvante pós-operatória de analgésicos/anti-inflamatórios entre os grupos estudados; e não houve correlação entre efeitos sistêmicos de medicações analgésicas/anti-inflamatórias adjuvantes empregadas e os grupos estudados.

\section{Suporte Financeiro}

Não houve suporte financeiro de fontes públicas, comerciais, ou sem fins lucrativos.

\section{Conflito de Interesses}

Os autores declaram não haver conflito de interesses.

\section{Referências}

1 Rawal N. Postoperative pain relief using regional anaesthesia. Curr Anaesth Crit Care 2007;18(03):140-148

2 Alagol A, Calpur OU, Usar PS, Turan N, Pamukcu Z. Intraarticular analgesia after arthroscopic knee surgery: comparison of neostigmine, clonidine, tenoxicam, morphine and bupivacaine. Knee Surg Sports Traumatol Arthrosc 2005;13(08):658-663

3 Raja SN, Dickstein RE, Johnson CA. Comparison of postoperative analgesic effects of intraarticular bupivacaine and morphine following arthroscopic knee surgery. Anesthesiology 1992;77 (06):1143-1147

4 Sun QB, Liu SD, Meng QJ, Qu HZ, Zhang Z. Single administration of intra-articular bupivacaine in arthroscopic knee surgery: a systematic review and meta-analysis. BMC Musculoskelet Disord $2015 ; 16: 21$

5 Wewers ME, Lowe NK. A critical review of visual analogue scales in the measurement of clinical phenomena. Res Nurs Health 1990;13(04):227-236

6 Schulz KF, Altman DG, Moher DCONSORT Group. CONSORT 2010 statement: updated guidelines for reporting parallel group randomized trials. Ann Intern Med 2010;152(11):726-732

7 Koh IJ, Chang CB, Seo ES, Kim SJ, Seong SC, Kim TK. Pain management by periarticular multimodal drug injection after anterior cruciate ligament reconstruction: a randomized, controlled study. Arthroscopy 2012;28(05):649-657

8 Osborne D, Keene G. Pain relief after arthroscopic surgery of the knee: a prospective, randomized, and blinded assessment of bupivacaine and bupivacaine with adrenaline. Arthroscopy 1993;9(02):177-180

9 Ng HP, Nordström U, Axelsson K, et al. Efficacy of intra-articular bupivacaine, ropivacaine, or a combination of ropivacaine, morphine, and ketorolac on postoperative pain relief after ambulatory arthroscopic knee surgery: a randomized double-blind study. Reg Anesth Pain Med 2006;31(01):26-33 
10 White AP, Laurent S, Wilkinson DJ. Intra-articular and subcutaneous prilocaine with adrenaline for pain relief in day case arthroscopy of the knee joint. Ann R Coll Surg Engl 1990;72 (06):350-352

11 Zeidan A, Kassem R, Nahleh N, et al. Intraarticular tramadolbupivacaine combination prolongs the duration of postoperative analgesia after outpatient arthroscopic knee surgery. Anesth Analg 2008;107(01):292-299

12 Kristensen PK, Pfeiffer-Jensen M, Storm JO, Thillemann TM. Local infiltration analgesia is comparable to femoral nerve block after anterior cruciate ligament reconstruction with hamstring tendon graft: a randomised controlled trial. Knee Surg Sports Traumatol Arthrosc 2014;22(02):317-323

13 Rokhtabnak F, Ale Bouyeh MR, Seyed Siamdust A, Masoomshahi M, Aghajani M. Comparison of the effects of intra-articular sole ropivacaine and combined ketorolac and ropivacaine for pain control after knee arthroscopy surgery. Br J Pain 2015;9(03): 149-156

14 Jazayeri SM, Mosaffa F, Abbasian M, Hosseinzadeh HR. Comparing the efficacy of intra-articular application of morphine and tramadol on postoperative pain after arthroscopic knee surgery. Anesth Pain Med 2012;2(01):28-31

15 Dragoo JL, Korotkova T, Kim HJ, Jagadish A. Chondrotoxicity of low $\mathrm{pH}$, epinephrine, and preservatives found in local anesthetics containing epinephrine. Am J Sports Med 2010;38(06): 1154-1159

16 Danieli MV, Cavazzani Neto A, Herrera PA. Intra-articular bupivacaine or bupivacaine and morphine after ACL reconstruction. Acta Ortop Bras 2012;20(05):258-261
17 Souza RH, Issy AM, Sakata RK. Analgesia intra-articular com morfina, bupivacaína ou fentanil após operação de joelho por videoartroscopia. Rev Bras Anestesiol 2002;52(05):570-580

18 Williams BA, Vogt MT, Kentor ML, Figallo CM, Kelly MD, Williams JP. Nausea and vomiting after outpatient ACL reconstruction with regional anesthesia: are lumbar plexus blocks a risk factor? J Clin Anesth 2004;16(04):276-281

19 Dal D, Tetik O, Altunkaya H, Tetik O, Doral MN. The efficacy of intra-articular ketamine for postoperative analgesia in outpatient arthroscopic surgery. Arthroscopy 2004;20(03):300-305

20 Goodwin RC, Amjadi F, Parker RD. Short-term analgesic effects of intra-articular injections after knee arthroscopy. Arthroscopy 2005;21(03):307-312

21 Eroglu A, Saracoglu S, Erturk E, Kosucu M, Kerimoglu S. A comparison of intraarticular morphine and bupivacaine for pain control and outpatient status after an arthroscopic knee surgery under a low dose of spinal anaesthesia. Knee Surg Sports Traumatol Arthrosc 2010;18(11):1487-1495

22 Wei J, Yang HB, Qin JB, Kong FJ, Yang TB. Single-dose intraarticular bupivacaine after knee arthroscopic surgery: a metaanalysis of randomized placebo-controlled studies. Knee Surg Sports Traumatol Arthrosc 2014;22(07):1517-1528

23 Zhou Y, Yang TB, Wei J, et al. Single-dose intra-articular ropivacaine after arthroscopic knee surgery decreases post-operative pain without increasing side effects: a systematic review and meta-analysis. Knee Surg Sports Traumatol Arthrosc 2016;24(05):1651-1659

24 Iwasaki K, Sudo H, Kasahara Y, et al. Effects of Multiple Intraarticular Injections of 0.5\% Bupivacaine on Normal and Osteoarthritic Joints in Rats. Arthroscopy 2016;32(10):2026-2036 